International Journal of Modern Physics A

(C) World Scientific Publishing Company

ANL-PHY-11578-TH-2006

\title{
Meson spectroscopy and properties using Dyson-Schwinger equations
}

\author{
A. KRASSNIGG \\ Institut für Physik, University of Graz, Universitätsplatz 5 \\ A-8010 Graz, Austria \\ andreas.krassnigg@uni-graz.at \\ C. D. ROBERTS and S. V. WRIGHT \\ Physics Division, Argonne National Laboratory, 97000 South Cass Avenue \\ Argonne, IL 60439, USA \\ cdroberts@anl.gov and svwright@anl.gov \\ Received 15082006 \\ Revised Day Month Year
}

\begin{abstract}
We study pseudoscalar and scalar mesons using a practical and symmetry preserving truncation of QCD's Dyson-Schwinger equations. We investigate and compare properties of ground and radially excited meson states. In addition to exact results for radial meson excitations we also present results for meson masses and decay constants from the chiral limit up to the charm-quark mass, e.g., the mass of the $\chi_{c 0}(2 P)$ meson.

Keywords: Dyson-Schwinger equations; Bethe-Salpeter equation; meson radial excitations.

PACS numbers:,12.38.Lg, 12.40.Yx,11.10.St
\end{abstract}

\section{Introduction}

Dyson-Schwinger equations (DSEs) are a nonperturbative continuum approach to quantum chromodynamics (QCD)! 1 They provide a means to study properties of the Green functions of $\mathrm{QCD} 23$ as well as hadrons as bound states of quarks and gluons. $\frac{456]}{516}$ Hadrons are studied in this framework of infinitely many coupled integral equations with the help of a symmetry preserving truncation scheme. In the case of mesons discussed here, one solves the Bethe-Salpeter equation (BSE) for a quark-antiquark pair. For calculations of baryon properties, e. g. electromagnetic, weak, and pionic form factors 7891011 one uses a covariant set of Faddeev equations! 71213

The Dyson-Schwinger equation framework has numerous features, amongst them: first, it is a Poincaré-covariant framework and thus ideally suited for the study of hadron observables such as, e.g., electromagnetic form factors. Secondly, symmetries are represented by Ward-Takahashi or Slavnov-Taylor identities, which are then built into the scheme used to truncate the infinite tower of coupled integral 
equations. If a truncation scheme respects such an identity at every step, then one can i) prove exact (model-independent) results and ii) use sophisticated models to calculate physical quantities which illustrate these results and automatically reflect the properties of the corresponding symmetry.

One such truncation is the so-called rainbow-ladder truncation, which has been used extensively and successfully to study meson ground states for more than a decade (see, e.g., Refs. 14, 15, 16, 17, 18). Despite this success it has become obvious that certain states and phenomena, such as axial-vector mesons, exotic mesons, ${ }^{\mathrm{a}}$ or heavy-light systems are not well-described in the most sophisticated calculations available to date. As a consequence, efforts are being made to go beyond this truncation, 1920212223 but these efforts are considerable, forcing present sophisticated calculations to remain at an exploratory stage.

\section{QCD Gap and Bethe-Salpeter Equations}

The homogeneous BSE is ${ }^{b}$

$$
[\Gamma(p ; P)]_{t u}=\int_{q}^{\Lambda}[\chi(q ; P)]_{s r} K_{r s}^{t u}(p, q ; P),
$$

where $p$ is the relative and $P$ the total momentum of the constituents, $r, \ldots, u$ represent color, Dirac and flavor indices,

$$
\chi(q ; P)=S\left(q_{+}\right) \Gamma(q ; P) S\left(q_{-}\right),
$$

$q_{ \pm}=q \pm P / 2$, and $\int_{q}^{\Lambda}$ represents a Poincaré invariant regularization of the integral, with $\Lambda$ the regularization mass-scale. In Eq. (11) $S$ is the renormalized dressed-quark propagator and $K$ is the fully amputated dressed-quark-antiquark scattering kernel; for details, see Refs. 14, 15

The dressed-quark propagator appearing in the BSE's kernel is determined by the renormalized gap equation

$$
\begin{aligned}
S(p)^{-1} & =Z_{2}\left(i \gamma \cdot p+m^{\mathrm{bm}}\right)+\Sigma(p), \\
\Sigma(p) & =Z_{1} \int_{q}^{\Lambda} g^{2} D_{\mu \nu}(p-q) \frac{\lambda^{a}}{2} \gamma_{\mu} S(q) \Gamma_{\nu}^{a}(q, p),
\end{aligned}
$$

where $D_{\mu \nu}$ is the dressed gluon propagator, $\Gamma_{\nu}(q, p)$ is the dressed quark-gluon vertex, and $m^{\mathrm{bm}}$ is the $\Lambda$-dependent current-quark bare mass. The quark-gluonvertex and quark wave function renormalization constants, $Z_{1,2}\left(\zeta^{2}, \Lambda^{2}\right)$, depend on the gauge parameter, the renormalization point, $\zeta$, and the regularization massscale. The leptonic decay constant of a pseudoscalar meson is calculated from the

"We use the term "exotic" to characterize mesons with quantum numbers that a system composed of a constituent-quark and constituent antiquark cannot have.

${ }^{\mathrm{b}}$ We employ a Euclidean metric, with: $\left\{\gamma_{\mu}, \gamma_{\nu}\right\}=2 \delta_{\mu \nu} ; \gamma_{\mu}^{\dagger}=\gamma_{\mu} ; \gamma_{5}=-\gamma_{1} \gamma_{2} \gamma_{3} \gamma_{4}$; $\operatorname{tr} \gamma_{5} \gamma_{\mu} \gamma_{\nu} \gamma_{\rho} \gamma_{\sigma}=-4 \varepsilon_{\mu \nu \rho \sigma}$; and $a \cdot b=\sum_{i=1}^{4} a_{i} b_{i}$. For a timelike vector $P_{\mu}, P^{2}<0$. 
solution of Eq. (1) via

$$
f_{\mathrm{PS}} \delta^{i j} P_{\mu}=Z_{2} \operatorname{tr} \int_{q}^{\Lambda} \frac{1}{2} \tau^{i} \gamma_{5} \gamma_{\mu} \chi_{\mathrm{PS}}^{j}(q ; P),
$$

which is gauge invariant, and cutoff and renormalisation-point independent.

\subsection{Rainbow-ladder truncation}

The first step in the symmetry-preserving truncation scheme described in Refs. 19 , 21, 24, 25] is the rainbow approximation to the gap equation combined with a ladder truncation in the BSE. The interaction kernels of Eqs. (11) and (3) then take the form

$$
K_{r s}^{t u}(p, q ; P)=-4 \pi \alpha\left(Q^{2}\right) D_{\mu \nu}^{\mathrm{free}}(Q)\left[\gamma_{\mu} \frac{\lambda^{a}}{2}\right]_{t s}\left[\gamma_{\nu} \frac{\lambda^{a}}{2}\right]_{r u}
$$

and

$$
\Sigma(p)=\int_{q}^{\Lambda} 4 \pi \alpha\left(Q^{2}\right) D_{\mu \nu}^{\mathrm{free}}(Q) \frac{\lambda^{a}}{2} \gamma_{\mu} S(q) \frac{\lambda^{a}}{2} \gamma_{\nu}
$$

where $Q=p-q, D_{\mu \nu}^{\text {free }}(Q)$ is the free gauge boson propagator ${ }^{\mathrm{c}}$ and $\alpha\left(Q^{2}\right)$ is an effective running coupling. The ultraviolet behavior of this coupling can be taken from perturbative QCD, while in the infrared one makes an Ansatz with sufficient enhancement to correctly reproduce the phenomenology of dynamical symmetry breaking, i. e., enhancement of the quark mass function on a domain $p^{2} \lesssim 1 \mathrm{GeV}^{2}$ and correspondingly a correct value of the chiral condensate. Such an Ansatz is 1516

$$
\frac{4 \pi \alpha(s)}{s}=\frac{4 \pi^{2}}{\omega^{6}} D s \mathrm{e}^{-s / \omega^{2}}+\frac{8 \pi^{2} \gamma_{m}}{\ln \left[\tau+\left(1+s / \Lambda_{\mathrm{QCD}}^{2}\right)^{2}\right]} \mathcal{F}(s),
$$

with $\mathcal{F}(s)=\left[1-\exp \left(-s /\left[4 m_{t}^{2}\right]\right)\right] / s, m_{t}=0.5 \mathrm{GeV}, \ln (\tau+1)=2, \gamma_{m}=12 / 25$ and $\Lambda_{\mathrm{QCD}}=\Lambda_{M S}^{(4)}=0.234 \mathrm{GeV}$. The free parameters of this model are the range $\omega$ and the strength $D$. One feature of the model is a specific parameter dependence of ground-state meson masses and properties, namely that they remain constant over a range of $\omega$, if $\omega D=$ const. is satisfied ${ }^{[26}$ This results in a one-parameter model, where the free parameter $\omega$ is varied in the interval $[0.3,0.5] \mathrm{GeV}$. In a calculation, $\omega$ and $D$ as well as the current-quark masses are fixed to pion mass and decay constant as well as the chiral condensate. That being done, further results are predictions.

${ }^{\mathrm{c}}$ We use Landau gauge in all calculations. 


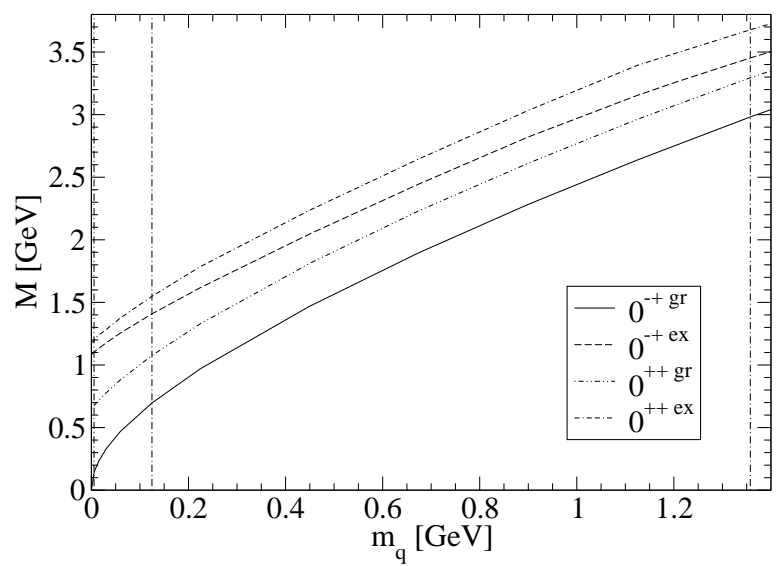

Fig. 1. Pseudoscalar and scalar meson masses for ground and excited states as functions of the current quark mass scaled to $1 \mathrm{GeV}$ by one-loop evolution. The three vertical dashed-dotted lines (the leftmost is very close to the $M$-axis) indicate the values for the $u / d, s$, and $c$ quark masses.

\subsection{Chiral symmetry}

One expression of the chiral properties of QCD is the axial-vector Ward-Takahashi identity

$$
P_{\mu} \Gamma_{5 \mu}^{j}(p ; P)=S^{-1}\left(p_{+}\right) i \gamma_{5} \frac{\tau^{j}}{2}+i \gamma_{5} \frac{\tau^{j}}{2} S^{-1}\left(p_{-}\right)-2 i m(\zeta) \Gamma_{5}^{j}(p ; P),
$$

which is written here for two quark flavors, each with the same current-quark mass: $\left\{\tau^{i}: i=1,2,3\right\}$ are flavor Pauli matrices. $\Gamma_{5 \mu}^{j}(k ; P)$ and $\Gamma_{5}^{j}(k ; P)$ are the axialvector and pseudoscalar vertices (for details, see Ref. 15). Equation (9) is satisfied by relating the kernels of the Bethe-Salpeter and gap equations (11) and (31), e.g., (6) and (7). A direct consequence of this identity is the relation

$$
f_{\mathrm{PS}} m_{\mathrm{PS}}^{2}=2 m(\zeta) \rho_{\mathrm{PS}}(\zeta),
$$

which relates the pseudoscalar meson mass $m_{\mathrm{PS}}$ and decay constant $f_{\mathrm{PS}}$ to the current quark mass $m(\zeta)$ and the residue of the pseudoscalar vertex at the pion pole $\rho_{\text {PS }}(\zeta)$ at the renormalization point $\zeta$. It has been shown ${ }^{27}$ that the implications of Eq. (10) in the chiral limit are different for the pion ground and excited states; namely, in the presence of dynamical chiral symmetry breaking the mass of the ground state vanishes, whereas for the excited states it is the leptonic decay constant that vanishes instead.

\section{Mesons}

\subsection{Meson ground states}

Ground state mesons and their properties have been studied in rainbow-ladder truncation using different Ansätze of various sophistication for the effective running coupling $15|16| 282930$ over a range of quark masses including the heavy-quark 


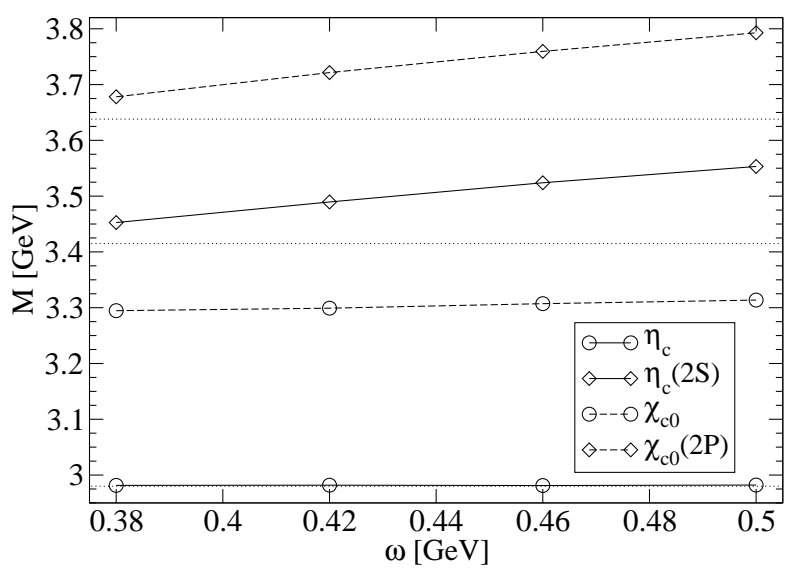

Fig. 2. Pseudoscalar and scalar $\bar{c} c$ meson masses as functions of the model parameter $\omega$. The three dotted lines correspond to the experimental values for the lower three states. The $\eta_{c}$ ground-state mass was fitted to the experimental value to fix the value of the charm-quark mass.

domain.21303132333435 The Ansatz of Ref. 16. Eq. (8), has been used successfully to calculate a large number of pseudoscalar and vector meson properties. We also used this Ansatz, since it has the correct ultraviolet behavior and thus yields reliable results not only for spectroscopy, but also for dynamical observables like form factors.

\subsection{Radial meson excitations}

It was natural to study radial excitations of pseudoscalar mesons first. An estimate of the excited pion mass and leptonic decay constant $[36$ and the structure of excitedstate Bethe-Salpeter amplitudes, which shows similar characteristics to a quantum mechanical wave function 37 were followed by more detailed studies of pseudoscalar meson radial excitations 27 and their electromagnetic properties 38 The present work includes a study of scalar mesons and their first radial excitations.

\subsection{Masses}

Figure 1 shows the ground and first radially excited states of pseudoscalar and scalar mesons as functions of the current-quark mass. Figs. 1 and 3 are generated from results for $\omega=0.38 \mathrm{GeV}$. In contrast to the ground states, the masses and properties of radial excitations do depend on the value of $\omega$, even if $\omega D=$ const. Since $r=1 / \omega$ corresponds to a range of the infrared part of the interaction, this means that radial meson excitations provide a means to study the long-range part of the strong interaction.

To obtain results independent of the choice for $\omega$, one can make use of ratios of calculated observables, which remain constant over a range of $\omega$, to estimate 


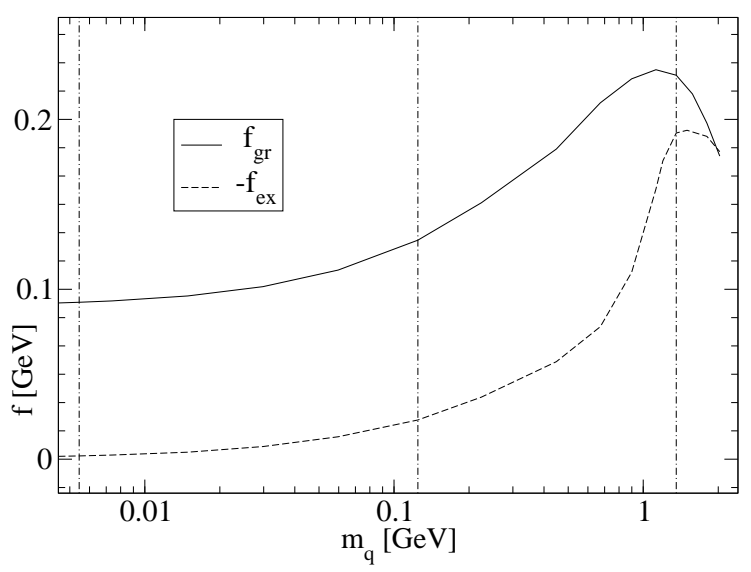

Fig. 3. $f_{\mathrm{PS}}$ for the ground- and $-f_{\mathrm{PS}}$ for the first radially excited-state pseudoscalar as functions of the current-quark mass scaled to $1 \mathrm{GeV}$ by one-loop evolution. The three vertical dashed-dotted lines correspond to the $u / d, s$, and $c$ quark masses. Estimated numerical errors are below $5 \%$.

properties of states on the basis of experimental values of other states that are known. An example for such an estimate is that of the mass of the $K(1460)$, the $K$ radial excitation, where the ratio $M_{K_{e x}} / M_{\pi_{e x}}$ is calculated to be 1.167 ; using the experimental number $M_{\pi_{e x}}=1.3 \pm 0.1 \mathrm{GeV}$ this yields $M_{K_{e x}}=1.52 \pm 0.12$ $\mathrm{GeV} \stackrel{39]}{ }$ Meanwhile, we have performed the analogous calculation for the leptonic decay constants of these states and found $f_{K_{e x}} / f_{\pi_{e x}} \simeq 10$ in agreement with an estimate via sum rules 4041

Figure 2 illustrates the same procedure to estimate the mass of the first scalar radial $\bar{c} c$ excitation $\chi_{c 0}(2 P)$. The ratio of the calculated masses for the $\chi_{c 0}(2 P)$ to the $\eta_{c}(2 S)$ is 1.066 . Via the experimental value of $3.64 \mathrm{GeV}$ (all experimental data are taken from Ref. 42) for the mass of the $\eta_{c}(2 S)$ we predict the $\chi_{c 0}(2 P)$ mass to be $3.88 \mathrm{GeV}$. This compares well to quark-model predictions, 43444546 which lie somewhat below the corresponding estimates from lattice QCD ${ }^{4748}$ We note that the $\chi_{c 0}(2 P)$ has not yet been observed experimentally.

\subsection{Decay constants}

For the pseudoscalar ground and first radially excited states we plot $f_{\mathrm{PS}}$ and $-f_{\mathrm{PS}}$, respectively, as functions of the current-quark mass in Fig. 3] While $f_{\mathrm{PS}}$ is not necessarily directly accessible experimentally for $\bar{Q} Q$ systems, where $Q$ is a heavyquark, it is always a well-defined axial-vector moment of the meson's Bethe-Salpeter amplitude. Its evolution with current-quark mass is therefore a useful tool with which to probe QCD and models thereof. One can see that both curves have a maximum at about the $c$-quark mass; for higher quark masses, the size of $f_{\mathrm{PS}}$ decreases for both ground and excited states. It is remarkable that this "turning point" occurs around the same quark mass as for the heavy-light case. 32 [35] At the 
Table 1. Values and ratios of $f_{\mathrm{PS}}$ for ground- and excited-state pseudoscalar mesons for $u / d, s$, and $c$ quark masses. Current-quark masses are given at the renormalization point $\zeta$ and scaled to $1 \mathrm{GeV}$ by one-loop evolution. All values in $\mathrm{GeV}$.

\begin{tabular}{cccccc}
\hline Quark & $m(\zeta=19 \mathrm{GeV})$ & $m(1 \mathrm{GeV})$ & $f_{\mathrm{gr}}$ & $-f_{\mathrm{ex}}$ & $-f_{\mathrm{ex}} / f_{\mathrm{gr}}$ \\
\hline$u / d$ & 0.0037 & 0.00545 & 0.092 & 0.0015 & 0.016 \\
$s$ & 0.0835 & 0.125 & 0.13 & 0.023 & 0.18 \\
$c$ & 0.905 & 1.357 & 0.23 & 0.19 & 0.83 \\
\hline
\end{tabular}

values for the $u / d-, s$-, and $c$-quark masses we extract the values for $f_{\mathrm{PS}}$ for the ground and $-f_{\mathrm{PS}}$ for excited states as well as their ratio. The results are summarized in Table 1. We note here that for the $s$ quark our pseudoscalar ground state does not correspond to an actual meson, since it consists merely of an $\bar{s} s$ component. However, a radial $\bar{s} s$ excitation can be identified with the $\eta(1475) \cdot \underline{39}$ Furthermore, the ground-state $\bar{s} s$ properties can also be studied on the lattice, where recent efforts have begun to study ratios of ground- and excited-state leptonic decay constants $\frac{49}{4}$

\section{Conclusions and Outlook}

We have extended previous studies of radial meson excitations by studying scalar excitations and quark masses up to the charm quark. While in the model we used ground-state meson properties do not depend on variations of the model parameter $\omega$, the specific parameter dependence of radial excitation properties allows investigations of the long-range part of the strong interaction between quarks. Without fixing $\omega$ to a particular value, we used ratios of properties of different excited states to make estimates for, e.g., the leptonic decay constant of the $K(1460)$ and the mass of the $\chi_{c 0}(2 P)$. This is made an efficacious procedure by the fact that ratios of excited-state properties remain constant over the domain of $\omega$ under investigation to a very good level of approximation. For both the ground and excited equal-mass pseudoscalar states we have calculated $f_{\mathrm{PS}}$ and observed a rise to a maximal size around the charm-quark mass and a decrease for higher quark masses. Further efforts in this direction will include studies of the radial excitations of vector mesons.

\section{Acknowledgments}

A. K. is grateful to his colleagues at the Physics Division of Argonne National Laboratory for their hospitality during a research visit, where part of this work was completed. We acknowledge useful discussions with M. S. Bhagwat and A. Höll. This work was supported by: the Austrian Science Fund FWF, SchrödingerRückkehrstipendium R50-N08; the Department of Energy, Office of Nuclear Physics, contract no. W-31-109-ENG-38; Helmholtz-Gemeinschaft Virtual Theory Institute VH-VI-041; and benefited from the facilities of ANL's Computing Resource Center.

\section{References}

1. C. D. Roberts and A. G. Williams, Prog. Part. Nucl. Phys. 33, 477 (1994). 
2. R. Alkofer and L. von Smekal, Phys. Rept. 353, 281 (2001).

3. C. S. Fischer, J. Phys. G 32, R253 (2006).

4. C. D. Roberts and S. M. Schmidt, Prog. Part. Nucl. Phys. 45, S1 (2000).

5. P. Maris and C. D. Roberts, Int. J. Mod. Phys. E 12, 297 (2003).

6. A. Höll, C. D. Roberts and S. V. Wright, arXiv:nucl-th/0601071

7. G. Hellstern, R. Alkofer, M. Oettel and H. Reinhardt, Nucl. Phys. A 627, 679 (1997).

8. J. C. R. Bloch, A. Krassnigg and C. D. Roberts, Few Body Syst. 33, 219 (2003).

9. R. Alkofer, A. Höll, M. Kloker, A. Krassnigg and C. D. Roberts, Few Body Syst. 37, 1 (2005).

10. A. Höll, R. Alkofer, M. Kloker, A. Krassnigg, C. D. Roberts and S. V. Wright, Nucl. Phys. A 755, 298 (2005).

11. A. Höll, C. D. Roberts and S. V. Wright, arXiv:nucl-th/0604029

12. M. Oettel, G. Hellstern, R. Alkofer and H. Reinhardt, Phys. Rev. C 58, 2459 (1998).

13. M. Oettel, M. Pichowsky and L. von Smekal, Eur. Phys. J. A 8, 251 (2000).

14. P. Maris, C. D. Roberts and P. C. Tandy, Phys. Lett. B 420, 267 (1998).

15. P. Maris and C. D. Roberts, Phys. Rev. C 56, 3369 (1997).

16. P. Maris and P. C. Tandy, Phys. Rev. C 60, 055214 (1999).

17. P. Maris and P. C. Tandy, Phys. Rev. C 61, 045202 (2000).

18. P. Maris and P. C. Tandy, Phys. Rev. C 62, 055204 (2000).

19. A. Bender, C. D. Roberts and L. Von Smekal, Phys. Lett. B 380, 7 (1996).

20. M. S. Bhagwat, M. A. Pichowsky, C. D. Roberts and P. C. Tandy, Phys. Rev. C 68, 015203 (2003).

21. M. S. Bhagwat, A. Höll, A. Krassnigg, C. D. Roberts and P. C. Tandy, Phys. Rev. C 70, 035205 (2004).

22. P. Watson, W. Cassing and P. C. Tandy, Few Body Syst. 35, 129 (2004).

23. C. S. Fischer, R. Alkofer, W. Cassing, F. Llanes-Estrada and P. Watson, Nucl. Phys. Proc. Suppl. 153, 90 (2006).

24. H. J. Munczek, Phys. Rev. D 52, 4736 (1995).

25. A. Bender, W. Detmold, C. D. Roberts and A. W. Thomas, Phys. Rev. C 65, 065203 (2002).

26. P. Maris, A. Raya, C. D. Roberts and S. M. Schmidt, Eur. Phys. J. A 18, 231 (2003).

27. A. Höll, A. Krassnigg and C. D. Roberts, Phys. Rev. C 70, 042203 (2004).

28. H. J. Munczek and A. M. Nemirovsky, Phys. Rev. D 28, 181 (1983).

29. H. J. Munczek and P. Jain, Phys. Rev. D 46, 438 (1992).

30. R. Alkofer, P. Watson and H. Weigel, Phys. Rev. D 65, 094026 (2002).

31. A. Krassnigg and P. Maris, J. Phys. Conf. Ser. 9, 153 (2005).

32. P. Maris and P. C. Tandy, arXiv:nucl-th/0511017

33. M. A. Ivanov, Y. L. Kalinovsky, P. Maris and C. D. Roberts, Phys. Lett. B 416, 29 (1998).

34. M. A. Ivanov, Y. L. Kalinovsky, P. Maris and C. D. Roberts, Phys. Rev. C 57, 1991 (1998).

35. M. A. Ivanov, Y. L. Kalinovsky and C. D. Roberts, Phys. Rev. D 60, 034018 (1999).

36. A. Krassnigg and C. D. Roberts, Fizika B 13, 143 (2004).

37. A. Krassnigg and C. D. Roberts, Nucl. Phys. A 737, 7 (2004).

38. A. Höll, A. Krassnigg, P. Maris, C. D. Roberts and S. V. Wright, Phys. Rev. C 71, 065204 (2005).

39. A. Höll, A. Krassnigg, C. D. Roberts and S. V. Wright, Int. J. Mod. Phys. A 20, 1778 (2005).

40. K. Maltman and J. Kambor, Phys. Rev. D 64, 093014 (2001).

41. K. Maltman and J. Kambor, Phys. Rev. D 65, 074013 (2002). 
42. PDG (W.-M. Yao et al.), J. Phys. G 33, 1 (2006).

43. S. Godfrey and N. Isgur, Phys. Rev. D 32, 189 (1985).

44. D. Ebert, R. N. Faustov and V. O. Galkin, Phys. Rev. D 67, 014027 (2003).

45. T. Barnes, S. Godfrey and E. S. Swanson, Phys. Rev. D 72, 054026 (2005).

46. O. Lakhina and E. S. Swanson, Phys. Rev. D 74, 014012 (2006).

47. CP-PACS Collaboration (M. Okamoto et al.), Phys. Rev. D 65, 094508 (2002).

48. P. Chen, Phys. Rev. D 64, 034509 (2001).

49. UKQCD Collaboration (C. McNeile and C. Michael), arXiv:hep-lat/0607032 